\title{
Alcohol induced region-dependent alterations of hemodynamic response: implications for the statistical interpretation of pharmacological fMRI studies
}

\author{
M. Luchtmann • K. Jachau • C. Tempelmann • \\ J. Bernarding
}

Received: 6 July 2009/Accepted: 21 April 2010/Published online: 26 May 2010

(c) The Author(s) 2010. This article is published with open access at Springerlink.com

\begin{abstract}
Worldwide, ethanol abuse causes thousands of fatal accidents annually as well as innumerable social dysfunctions and severe medical disorders. Yet, few studies have used the blood oxygenation level dependent functional magnetic resonance imaging method (BOLD fMRI) to map how alcohol alters brain functions, as fMRI relies on neurovascular coupling, which may change due to the vasoactive properties of alcohol. We monitored the hemodynamic response function (HRF) with a high temporal resolution. In both motor cortices and the visual cortex, alcohol prolonged the time course of the HRF, indicating an overall slow-down of neurovascular coupling rather than an isolated reduction in neuronal activity. However, in the supplementary motor area, alcoholinduced changes to the HRF suggest a reduced neuronal activation. This may explain why initiating and coordinating complex movements, including speech production, are often impaired earlier than executing basic motor patterns. Furthermore, the present study revealed a potential
\end{abstract}

\footnotetext{
M. Luchtmann $(\bowtie) \cdot$ J. Bernarding $(\bowtie)$

Institute for Biometry and Medical Informatics,

Otto-von-Guericke University of Magdeburg,

Leipziger Str. 44, 39120, Magdeburg, Germany

e-mail: michel.luchtmann@med.ovgu.de

J. Bernarding

e-mail: johannes.bernarding@med.ovgu.de

K. Jachau

Institute of Forensic Medicine, Otto-von-Guericke University of

Magdeburg, Leipziger Str. 44, 39120, Magdeburg, Germany

e-mail: katja.jachau@med.ovgu.de

C. Tempelmann

Department of Neurology, Otto-von-Guericke University of

Magdeburg, Leipziger Str. 44, 39120, Magdeburg, Germany

e-mail: claus.tempelmann@med.ovgu.de
}

pitfall associated with the statistical interpretation of pharmacological fMRI studies based on the general linear model: if the functional form of the HRF is changed between the conditions data may be erroneously interpreted as increased or decreased neuronal activation. Thus, our study not only presents an additional key to how alcohol affects the network of brain functions but also implies that potential changes to neurovascular coupling have to be taken into account when interpreting BOLD fMRI. Therefore, measuring individual drug-induced HRF changes is recommended for pharmacological fMRI.

Keywords Alcohol · Ethanol · Functional MRI ·

Pharmacological fMRI - Hemodynamic response function . BOLD effect $\cdot$ Statistical parametric mapping

\section{Introduction}

Alcohol is the most frequently used psychoactive drug worldwide. Social behavior, perception, and motor output are usually altered significantly after ethanol intake. Longterm misuse may lead to addiction and ultimately results in severe neurological, medical, and social disorders. Although somatic and psychological effects have been investigated extensively, the direct time and spatial resolved influence of alcohol on neuronal functions is less understood. Likewise it remains unclear how and to what degree different functional networks in the brain are impaired at even moderate alcohol levels: Motor functions such as walking or grasping may remain intact, but speech production, mental calculation, or social behavior may already be severely affected (Hindmarch et al. 1991; Moskowitz et al. 1985).

Ethanol as the active ingredient of consumable alcohol induces changes to brain functions that have been 
monitored non-invasively using electroencephalography, positron emission tomography, single photon emission computed tomography, or transcranial magnetic stimulation (Fonseca et al. 2001; Kaehkoenen et al. 2003; Lewis et al. 1970; Sano et al. 1993; Volkow et al. 1990, 1988; Wendt and Risberg 2001). BOLD fMRI (Ogawa et al. 1990) has been used in numerous studies, but rarely to monitor ethanol-induced activation changes such as in the visual system (Levin et al. 1998), in the auditory system (Seifritz et al. 2000), or in extended cortical networks (Calhoun et al. 2004a, b; Meda et al. 2009; Van Horn et al. 2006). One reason may be the vasoactive effects of ethanol, which may compromise basic mechanisms underlying BOLD imaging (Friston et al. 1995; Ogawa et al. 1990; Villringer and Dirnagl 1995): Neuronal activation increases the regional cerebral blood flow ( $\mathrm{rCBF})$ and blood volume (rCBV) via neurovascular coupling, and this complex interplay between inflowing and outflowing oxygenated and deoxygenated hemoglobin leads to the so-called hemodynamic response function (HRF) that can be monitored with appropriate MR sequences (BOLD imaging; Buxton et al. 1998; Ogawa et al. 1990; Stephan et al. 2007; Villringer and Dirnagl 1995). Ethanol may affect this BOLD contrast in multiple ways. A potential ethanol-induced increase in $2-10 \%$ of the global CBF (Volkow et al. 1988) should still enable detection of BOLD signals, although the BOLD signal would be decreased by about $3-15 \%$. Seifritz et al. (2000) also found a $12-27 \%$ ethanol-influenced activation decrease in the acoustic cortex, whereas Levin et al. (1998) measured a 33\% activation decrease in the visual cortex after ethanol ingestion. BOLD fMRI of ethanol-induced alterations to neuronal activity should therefore be possible as long as the cerebral vessels have sufficient dilatation reserve. However, drugs may not only reduce or amplify neuronal activation but also change the dynamics of the complex neurovascular coupling leading to altered time courses of the HRF. Information about potential drug-induced changes of the time course of the HRF is sparse (Breiter et al. 1997), and no such information is available yet for alcohol. Therefore, (a) we monitored the time course of the HRF with a time resolution of $500 \mathrm{~ms}$, (b) we studied whether the time course of the HRF is altered even after moderate alcohol ingestion, and (c) whether this alteration is specific to the cortical region.

As the BOLD signal is often small and noisy, a statistical data analysis is required in most cases. A statistical design using a placebo-controlled study imposes the problem that experienced social drinkers can discriminate, as a result of the presence and absence of interoceptive effects, between alcoholic beverages and the corresponding placebo drink. Hammersley et al. (1992) and Glautier et al. (1992) emphasized that placebos may be only suitable to mimic modest alcohol doses which may not be representative for social drinking, and which may lack induce effects usually ascribed to alcohol. This view is supported by other studies that showed that placebos of alcoholic beverages are not suitable for studies using examining blood alcohol concentrations above $0.4 \%$ (Martin et al. 1990). Furthermore, the HRFs vary inter-individually quite strongly (Aguirre et al. 1998) making it difficult to compare the time courses of the HRF between different subjects. We therefore preferred to apply a test/retest design as this would enable to monitor directly the individual alcohol-induced changes of the HRF. But additional attention has to be paid when interpreting pharmacological fMRI data. fMRI data are usually analyzed by using a General Linear Model (GLM), where standard regressors modeling the HRF are applied to infer the signal course of the measured data (Friston et al. 2006). In most studies of pharmacological fMRI it is assumed that only the amplitude of the HRF is changing, while the underlying functional form of the HRF remains unchanged despite potential effects of experimental conditions or applied drugs on neurovascular coupling mechanisms. However, if the HRF changes its form considerably, this in turn may lead to false positive or false negative activation changes depending how the time course of the changed HRF correlates with this canonical regressor. In our study, we therefore used a hybrid data analysis strategy: after determining the regions of neuronal activation using a standard GLM, we extracted the data and studied direct changes to the HRF in a straight-forward way based on the averaged measured data. This enabled additionally to test how alteration of the HRF may affect interpretation of the generally applied statistical analysis of fMRI data.

\section{Methods}

\section{Subjects}

Fourteen healthy right-handed volunteers $(7 \mathrm{~m}, 7 \mathrm{f}, 21-$ 29 years, mean $23.2 \pm 1.9$ years) were examined before and after administration of ethanol. All participants gave their written informed consent to the study, which was approved by the Local Ethics Committee of the University of Magdeburg in compliance with national legislation and the Code of Ethical Principles for Medical Research Involving Human Subjects of the World Medical Association (Declaration of Helsinki). All of them were social drinkers and had experience with higher doses of alcohol, but had no self-reported history of neurological disease, major psychiatric disturbance, substance abuse or psychoactive prescriptive medications usage. 
Study protocol

Prior to the MR examination, the volunteers ingested no alcohol for $24 \mathrm{~h}$ and refrained from eating for $6 \mathrm{~h}$. Each person underwent BOLD imaging with identical tasks before and after ethanol administration. To minimize potential training effects, each person was required to perform the task twice prior to the measurement. We chose a test/retest design by using an identical set of on/off tasks before and after administration of the drug (Breiter et al. 1997; Chen et al. 1997; Stein et al. 1998) as individual hemodynamic response functions vary considerably (Aguirre et al. 1998; Handwerker et al. 2004). The runs of the session before ethanol administration served as the baseline, that is, each person served as a control for the individual ethanol effect. After the first examination each volunteer left the scanner and ingested a drink containing pure ethanol mixed with orange juice to a total of about $400 \mathrm{ml}$. The amount of ethanol $a$ was estimated based on modified Widmark's equation (Seidl et al. 2000) to reach a maximum blood ethanol concentration (BAC, $C_{\mathrm{O}}$ in $\mathrm{mg} / \mathrm{g}$ ) of $1.0 \%$ :

$C_{\mathrm{O}}=\frac{a}{p \times r}$

where $p$ denotes the body weight in $\mathrm{kg}$ and $r$ denotes the individual correction factor according to the equations developed by Seidl et al. (2000).

After ethanol administration, the volunteers had to wait until their breath alcohol level reached about $80 \%$ of the intended BAC of $1 \%$. This time varied between 50 and $70 \mathrm{~min}$. The breath alcohol level was monitored with a legally certified breath alcohol test device (Draeger 7110 Evidential, Germany). It was assumed that the BAC reaches its approximate maximum at the beginning of the retest session and decreases slowly thereafter (Jones and Jonsson 1994; Levin et al. 1998). The influence of ethanol on the task performance can therefore be assumed to be constant during the second scan period (retest examination). Additionally, a small intravenous catheter in a cubital vein drained 10 blood samples every $15 \mathrm{~min}$ for a retrospective determination of the BAC using an alcohol dehydrogenase-based enzyme assay and a gas chromatography method. Retrospective mean BAC was $0.82 \pm 0.07 \%$.

\section{Experimental setting}

A visually paced paradigm served for the measurement of the individual regional HRFs in the visual cortex (VC), the supplementary motor area (SMA) as well as in the left and right motor cortex (LMC, RMC). The visual stimulus consisted of a flickering checkerboard presented for $1 \mathrm{~s}$ with a flicker-frequency of $8 \mathrm{~Hz}$. The stimuli were presented in an event-related design with a fixed interstimulus interval of $19 \mathrm{~s}$ leading to a total block length of $20 \mathrm{~s}(1 \mathrm{~s}$ checkerboard, 19 s black screen). The rather long interstimulus interval was required to monitor the HRF as completely as possible. A small centrally located visual fixation cross was presented throughout the complete block of $20 \mathrm{~s}$ to minimize eye movements. The color of this fixation-cross changed randomly from gray to blue for half a second. To keep attention high, volunteers had to count the number of blue crosses and reported this number after the complete run to the experimenter. The checkerboard served additionally to initiate an event-related motor task where the subjects touched sequentially with their fingers the opposing thumb as fast as possible for about $1 \mathrm{~s}$. To recognize training or habituation effects, all volunteers were asked to perform two complete runs of the paradigm prior to the scanning session first outside and then inside the scanner as well as to perform the task twice both in the pre- and post-ethanol run. For the statistical data analysis, this resulted in 4 runs $(2$ runs for sober, and 2 runs for ethanol condition), each of the runs containing 30 blocks of 20 s duration.

\section{MR Imaging}

All anatomical and fMRI data were acquired on a $3 \mathrm{~T}$ scanner (Magnetom Trio, Siemens, Erlangen, Germany) using a standard 8-channel phased array head coil. For anatomic data sets, we used a T1 weighted MPRAGEsequence $(\mathrm{FOV}=256 \mathrm{~mm}$, matrix size $=256 \times 256$, slices $=192$, slice thickness $=1 \mathrm{~mm}, \mathrm{TR}=2,500 \mathrm{~ms}$ ). To measure the individual HRF, a gradient echo EPI sequence was used with TR $=500 \mathrm{~ms}, \mathrm{TE}=30 \mathrm{~ms}, \mathrm{FOV}=192 \mathrm{~mm}$, matrix size $=64 \times 64,7$ oblique slices covering the VC, SMA, LMC, RMC. The high time resolution of $500 \mathrm{~ms}$ led to a maximum number of 7 slices. Thereof, the slice thickness was chosen to $6 \mathrm{~mm}$ (25\% distance factor) to allow acquiring a sufficient volume of the activated cortical areas.

Image processing and statistical analysis

The individual HRFs were analyzed in a multi-step procedure. Data were preprocessed and analyzed with SPM $^{1}$ running on Matlab2007b ${ }^{2}$. All images were realigned to the first saturated image of the fMRI data set, normalized into the standard stereotaxic space by using the MNI-template and smoothed with a 6-mm full-width-at-half-maximum Gaussian kernel. To reduce time drifts and high frequency

\footnotetext{
${ }^{1}$ FIL - SPM5 - Revision 946, http://www.fil.ion.ucl.ac.uk/spm/

2 MathWorks, Version 2007b, http://www.mathworks.com/
} 
noise in the data, a high-pass cut-off (128 s) and the SPM built-in AR(1) model were applied (Friston et al. 2006).

The regions of evoked brain activation under the preethanol conditions $\left(P<10^{-5}\right.$, FWE) were determined by convolving the stimulus duration (1 s) with the canonical SPM built-in hemodynamic response function. These regions served as regions of interest (ROI) in the subsequent pre- and post-ethanol time course analysis. The corresponding signal time courses were extracted and averaged for each subject across the ROIs and 60 blocks (two runs sober and two runs ethanol) using the toolbox Marsbar $^{3}$ yielding the individual HRFs for activated cortical regions RMC, LMC, SMA, VC. Subsequently, the individual HRFs were fitted with a triple gamma-variate function according to Eq. 2 using the LevenbergMarquardt algorithm. The triple gamma-variate function was required to obtain a good fit simultaneously to the initial dip, the positive overshoot, and the post-stimulus undershoot.

$$
\begin{aligned}
H R F\left(t, T_{i}, F_{i}, A_{i}\right) & =\sum_{i=1}^{3}(-1)^{i} A_{i} \times\left(\frac{t}{T_{i}}\right)^{\alpha_{i}} \times \exp \left(\frac{t-T_{i}}{-\beta_{i}}\right) \\
\alpha_{i} & =\frac{T_{i}^{2}}{F_{i}^{2}} \times 8 \times \log (2) \\
\beta_{i} & =\frac{F_{i}^{2} / T_{i}}{8 / \log (2)} \\
T & =\text { time to peak } \\
F & =\text { full width at half maximum } \\
A & =\text { amplitude }
\end{aligned}
$$

The fit with this analytic function yielded an increased time resolution and a subsequent optimized estimation of the parameters amplitude, time-to-peak (TTP), full width at half maximum (FWHM), and area-under-curve (AUC) of the positive overshoot. Potential changes to the baseline and the mentioned parameters after ethanol ingestion as well as the differences between the first and second run in each condition (sober and ethanol) and potential differences between the dominant and non-dominant hemisphere were tested using a 2-tailed paired $t$-test with an alpha-level of 5\%. All statistical analysis were conducted using $\operatorname{SPSS}^{4}$.

\section{Results}

The RMC, LMC, SMA, as well as the VC exhibited an increased BOLD signal against the rest condition in both

\footnotetext{
3 MarsBar-Team, Version 0.41, http://marsbar.sourceforge.net/

${ }^{4}$ SPSS, Version 15, http://www.spss.com/
}

the pre- and post-ethanol conditions. No significant change in the BAC was detected during experiment time. Baseline and corresponding HRFs of each region were averaged for each individual. No significant changes in the mean baseline were visible after ethanol ingestion (Table 1). However, to exclude potential signal changes caused by technical aspects, we solely analyzed changes of the individual HRFs relative to the mean baseline. The individual HRFs varied considerably, but all time courses were changed by the ingestion of ethanol, which, on average, led to a significantly decreased amplitude in each activated area (Fig. 1), with a mean reduction between 14.1 and $34.1 \%$ (Table 2). The most evident decrease was seen in the SMA. The FWHM showed a significant increase in the $\operatorname{RMC}(0.39 \pm 0.44 \mathrm{~s})$, the LMC $(0.38 \pm 0.55 \mathrm{~s})$, and the VC $(0.43 \pm 0.58 \mathrm{~s})$, whereas this effect was not significant in the SMA (Table 2). The post-stimulus undershoot shifted toward positive values. To estimate whether the reduced amplitude was balanced by an increased FWHM, the area under the curve (AUC) of the positive overshoot of the BOLD signal was determined. Although the AUC remained unchanged in the $\mathrm{RMC}$, LMC, and $\mathrm{VC}$, a significant decrease of $29.9 \%$ could be observed in the SMA after ingestion of ethanol. No significant ethanol-induced differences were found between dominant and non-dominant hemisphere ( $P$-values varied between 0.578 and 0.999 depending on amplitude, TTP, FWHM, and AUC) or between males and females ( $P$-values varied between 0.178 and 0.883 depending on the cortical region and the according parameters amplitude, TTP, FWHM, and AUC). Also, no habituation effects were found between the first and the second run in each of the pre- and post-ethanol condition (2-tailed paired $t$-test with an alpha-level of 5\%).

For comparison with a standard fMRI analysis Fig. 2 and Table 3 show the results of the random-effects analysis using SPM5. The contrast for the sober condition as well as the contrast for the ethanol condition exhibited strong activations in the observed areas LMC, RMC, SMA, and VC. Despite the apparent reduction of the amplitude, as seen in Fig. 1 and Table 2, the contrast for the difference of both conditions (sober-ethanol) shows no significant activated areas. Thus the results of the random-effects analysis using the SPM built-in hemodynamic response function as regressor leads to the conclusion that ethanol does not

Table 1 Statistical analysis of mean baseline-levels

\begin{tabular}{llccc}
\hline Region & Sober & Ethanol & Difference & $P$-value \\
\hline LMC & 233.89 & 236.52 & $2.63 \pm 14.66$ & 0.513 \\
RMC & 226.81 & 225.40 & $-1.41 \pm 13.84$ & 0.709 \\
SMA & 191.25 & 197.64 & $6.39 \pm 12.94$ & 0.087 \\
VC & 231.25 & 224.67 & $-6.59 \pm 6.59$ & 0.131 \\
\hline
\end{tabular}



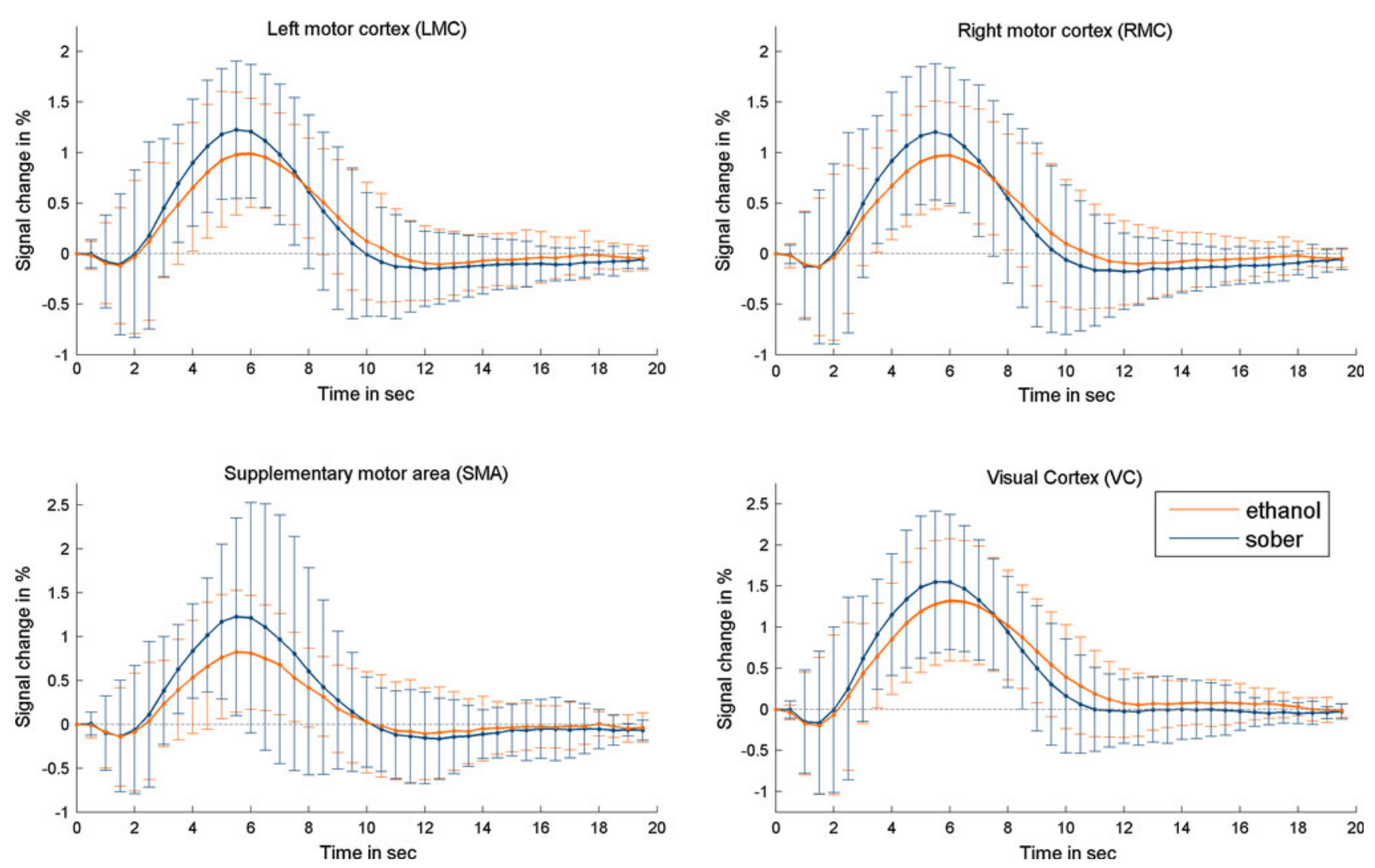

Fig. 1 Results of the mean raw data of the hemodynamic response functions (HRF) averaged across all subjects and within observed regions. To depict more clearly the time course of the HRF data points were connected by straight lines. All regions exhibit a reduced

interfere with the evoked cerebral activations in the observed regions LMC, RMC, SMA, and VC.

\section{Discussion}

To our knowledge, this is the first event-related fMRI study to the direct influence of ethanol on the time course of the hemodynamic response function (HRF). The influence of alcohol on brain metabolism is manifold and depends on multiple molecular mechanisms (Davies 2003; Fonseca et al. 2001; Learn et al. 2003; Little 1999; Seo and Rivier 2003; Volkow et al. 1990). In vivo monitoring of alcoholinduced changes of neuronal functions in humans requires non-invasive techniques such as EEG, MEG, PET, or MRI (Blinkenberg et al. 1996; Kaehkoenen et al. 2003; Lewis et al. 1970; Rhodes et al. 1975; Sano et al. 1993; Volkow et al. 1988; Wendt and Risberg 2001). While functional MRI based on the blood oxygenation changes (BOLD contrast) as a function of neuronal activity has been used in numerous studies (Horwitz et al. 2000) the application of BOLD techniques to alcohol-induced activation changes has been reported only by few groups (Calhoun et al.

amplitude (most pronounced in the SMA) as well as a shift of the undershoot toward positive values. Both motor cortices and the visual cortex exhibit also a prolonged FWHM (for details see Table 2)

2004a; Levin et al. 1998; Seifritz et al. 2000; Van Horn et al. 2006) where a 18-33\% signal decrease was found in the visual system (Levin et al. 1998) or the 22-27\% signal decrease found in the acoustic system (Seifritz et al. 2000). In a more complex task of driving in a virtual reality environment alcohol modulated specific networks indicating global and local effects (Calhoun et al. 2004a; Meda et al. 2009).

The main reason is the difficulty to account for the influence of alcohol on the brain metabolism as well as for the effects on the neurovascular coupling. This renders the interpretation of BOLD-technique-based data difficult and may obscure direct alcohol-induced alterations of the neuronal activity. Supplementary information is provided by PET studies. Alcohol consumption was shown to decrease the glucose metabolism (Volkow et al. 1990) indicating a partial uncoupling between global cerebral blood flow and cellular metabolism. The hypothesis that alcohol-induced uncoupling involves global rather than local effects seemed to be supported by studying the visual stimulation before and after alcohol consumption (Meda et al. 2009; Wendt et al. 1994). Other studies however (Calhoun et al. 2004a; Van Horn et al. 2006; Wendt and 
Table 2 Results of the statistical analysis of the parameters of the HRF fit according to Eq. 2

\begin{tabular}{|c|c|c|c|c|c|}
\hline & Sober & Ethanol & Difference & Diff. $\%$ & $P$-value \\
\hline \multicolumn{6}{|l|}{ Left motor cortex (LMC) } \\
\hline Amplitude & $3.00 \pm 0.64$ & $2.44 \pm 0.59$ & $-0.56 \pm 0.55$ & -18.67 & 0.002 \\
\hline Time to peak & $5.53 \pm 0.48$ & $5.99 \pm 0.69$ & $0.46 \pm 0.37$ & 8.32 & $<\mathbf{0 . 0 0 1}$ \\
\hline Full width at half maximum & $4.63 \pm 0.77$ & $5.01 \pm 0.77$ & $0.38 \pm 0.55$ & 8.21 & 0.024 \\
\hline Area under the curve & $13.92 \pm 3.18$ & $12.30 \pm 3.10$ & $-1.62 \pm 3.44$ & -11.64 & 0.102 \\
\hline \multicolumn{6}{|l|}{ Right motor cortex (RMC) } \\
\hline Amplitude & $2.92 \pm 0.68$ & $2.39 \pm 0.49$ & $-0.53 \pm 0.53$ & -18.15 & 0.002 \\
\hline Time to peak & $5.42 \pm 0.49$ & $5.89 \pm 0.72$ & $0.47 \pm 0.31$ & 8.67 & $<\mathbf{0 . 0 0 1}$ \\
\hline Full width at half maximum & $4.63 \pm 0.81$ & $5.02 \pm 0.78$ & $0.39 \pm 0.44$ & 8.42 & 0.006 \\
\hline Area under curve & $13.53 \pm 3.94$ & $12.15 \pm 3.05$ & $-1.38 \pm 2.46$ & -10.20 & 0.055 \\
\hline \multicolumn{6}{|c|}{ Supplementary motor area (SMA) } \\
\hline Amplitude & $3.02 \pm 1.35$ & $1.99 \pm 0.70$ & $-1.03 \pm 1.46$ & -34.11 & 0.021 \\
\hline Time to peak & $5.42 \pm 0.51$ & $5.80 \pm 0.54$ & $0.38 \pm 0.33$ & 7.01 & 0.001 \\
\hline Full width at half maximum & $4.48 \pm 0.83$ & $4.60 \pm 1.00$ & $0.12 \pm 0.90$ & 2.68 & 0.625 \\
\hline Area under the curve & $13.70 \pm 5.60$ & $9.61 \pm 3.46$ & $-4.09 \pm 6.55$ & -29.85 & $\mathbf{0 . 0 3 6}$ \\
\hline \multicolumn{6}{|l|}{ Visual cortex (VC) } \\
\hline Amplitude & $3.75 \pm 0.81$ & $3.22 \pm 0.71$ & $-0.53 \pm 0.44$ & -14.13 & 0.001 \\
\hline Time to peak & $5.71 \pm 0.42$ & $6.16 \pm 0.55$ & $0.45 \pm 0.36$ & 7.88 & $<0.001$ \\
\hline Full width at half maximum & $5.04 \pm 0.65$ & $5.47 \pm 0.65$ & $0.43 \pm 0.58$ & 8.53 & 0.016 \\
\hline Area under the curve & $19.24 \pm 4.31$ & $18.77 \pm 5.46$ & $-0.47 \pm 6.55$ & -2.44 & 0.643 \\
\hline
\end{tabular}

Significant results (alpha level $<5 \%$ ) are depicted bold face

Risberg 2001) indicated local differences of the influence of alcohol on brain metabolism.

Using PET several groups determined an alcoholinduced vasodilatation between 2\% (Volkow et al. 1988) and $12 \%$ (Sano et al. 1993) at BAC comparable to our studies. To estimate whether alcohol-induced alterations of neurovascular responses would still be detectable by BOLD imaging model Seifritz et al. (2000) performed model calculations. They concluded that a reduction of the BOLD signal of about $15 \%$ would be expected assuming an increase in the rest CBF of 10\%. BOLD imaging should therefore be feasible if after moderate alcohol intake the cerebral vessels still have the capacity to react appropriately on increased neuronal activity with a net increase large enough to be detected by BOLD imaging.

Additional experiments investigating the regional neuronal activations under different vascular conditions support this assumption. Shimosegawa et al. (1995) investigated the rCBF response after visual stimulation under hypo-, normoand hypercapnia. Changing from hypocapnia to hypercapnia which also leads to vasodilatation they found an increasing regional and global CBF. But in all tasks the changes relative to the rest condition remained unchanged with and without hypercapnia. This might be interpreted that despite an increased CBF the vascular reserve is still not exhausted and provides enough capacity for the neurovascular response required by the increased focal neuronal activity.
Another study showed that CBF responses on external stimuli were reduced under application of the vasodilating drug acetazolamide (Bruhn et al. 1994). The findings were explained by the reduced capability of already strongly dilated vessels to further dilate. Other studies however indicate an azetazolamide-induced inhibition of neuronal activity (Oishi et al. 1995). Whether the studied effects are due to a suppression of neuronal activity or caused by a reduced capacity of regional vasodilatation (or most probable both effects) is not yet completely understood.

All drugs that influence vessel properties as well as the cerebral metabolism might show direct effects on the HRF. As the HRF serves as the measurement parameter for the neurovascular coupling in fMRI, the analysis of HRF changes should give direct insight into possible impairments of the processes involved.

The study confirms that neurovascular coupling underlying the BOLD effect can be detected at moderate ethanol levels, supporting the assumption that cerebral vessels still have sufficient dilating capacity (Levin et al. 1998; Seifritz et al. 2000; Volkow et al. 1990)—similar to with other vasoactive drugs (Bruhn et al. 1994; Shimosegawa et al. 1995). As no habituation effects were observed within both the sober and ethanol condition, the signal changes should be caused mainly by ethanol ingestion.

Concerning the regional differences, it has been shown that even for sober conditions, BOLD signals vary locally 


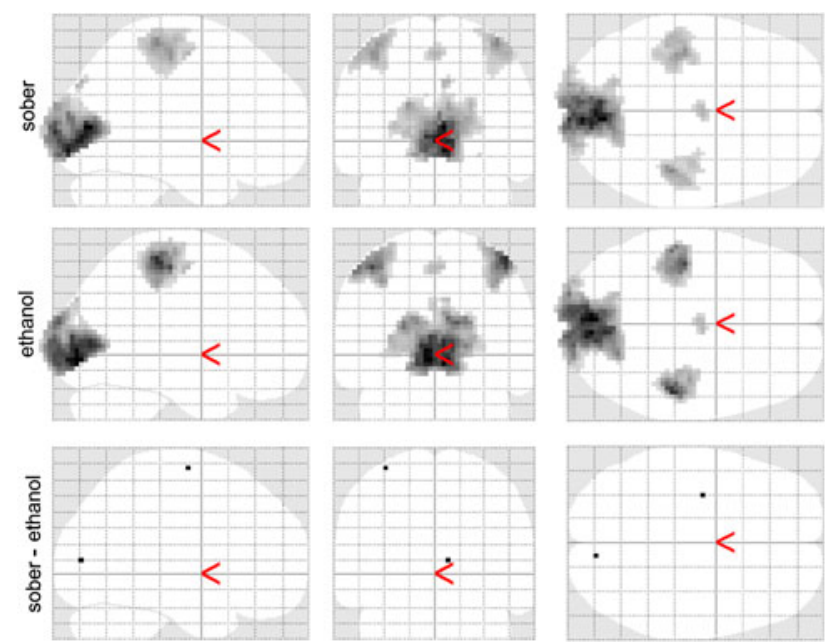

Fig. 2 Results of the random effects analysis of all 14 subjects. Activation-maps of the 2nd-level analysis across all subjects using SPM (FDR corr. $-P<0.05$ ). Despite the decrease of the the mean amplitude of the hemodynamic responses in the observed areas LMC, RMC, SMA, and VC (see Fig. 1) the 2nd-level analysis using a general linear model depicted only two significant voxels for the difference contrast sober versus ethanol

Table 3 Statistical values of the random effects analysis using SPM

\begin{tabular}{llllllll}
\hline Contrast & Region & \multicolumn{2}{c}{ MNI coordinates } & t val. & ext. voxel & vol. $\left(\mathrm{cm}^{3}\right)$ \\
\cline { 3 - 5 } & & $x$ & $y$ & $z$ & & & \\
\hline Sober & LMC & 42 & -30 & 63 & 7.67 & 263 & 2.37 \\
& RMC & -42 & -21 & 57 & 7.11 & 298 & 2.68 \\
& SMA & 0 & -9 & 57 & 5.18 & 32 & 0.29 \\
& VC & 6 & -75 & 9 & 15.95 & 1,339 & 12.05 \\
Ethanol & LMC & 48 & -30 & 63 & 10.83 & 286 & 2.57 \\
& RMC & -42 & -21 & 57 & 9.79 & 322 & 2.90 \\
& SMA & 3 & -9 & 57 & 4.51 & 29 & 0.26 \\
& VC & -3 & -84 & -3 & 13.44 & 1,443 & 12.99 \\
Sober & LMC & - & - & - & - & - & - \\
& RMC & -33 & -9 & 69 & 3.94 & 1 & 0.26 \\
Ethanol & SMA & - & - & - & - & - & - \\
& VC & 9 & -81 & 9 & 3.89 & 1 & 0.26 \\
\hline
\end{tabular}

(Aguirre et al. 1998; Handwerker et al. 2004), and BOLD responses in the SMA differ from those in the RMC and LMC (Obata et al. 2004). Unsurprisingly, ethanol aggravates these regional differences: In the RMC, LMC, and $\mathrm{VC}$, the decreased amplitude was counteracted by an increased FWHM, leading to an unchanged AUC; in the SMA, the amplitude decreased strongly but the FWHM remained unchanged, leading to a reduced AUC.

This points to different sensitivities of brain functions to ethanol. The quasi-automatic movement pattern as well as the simple visual task seemed to activate the neuronal population to the same degree as in the pre-ethanol condition but the entire system appeared to work more slowly (increased TTP and enlarged FWHM). In contrast, the strong reduction of the AUC in the SMA indicates an earlier and more severe impairment of neurovascular coupling as well as a potentially stronger reduction in neuronal activity. The SMA being important for complex functions such as movement initiation or speech (Chung et al. 2005), these higher functions may be impaired earlier than basic motor functions responsible for simple repetitive movement patterns. In the balloon model (Buxton et al. 1998; Friston et al. 2000; Stephan et al. 2007) it was shown that a isolated reduction of the neuronal efficacy resulted only in a reduced amplitude without changing the form of the HRF as seen in the SMA in our study. The prolonged time course seen in the motor cortices and the visual cortex require additional parameter changes. Although the parameter inter-dependencies of the BOLD signal are very complex prolongation of the transit time or change of the autoregulation may explain the observed alteration of the HRF (Friston et al. 2000).

Some groups reported a dominant effect of alcohol on the right hemisphere (Levin et al. 1998; Rhodes et al. 1975; Seifritz et al. 2000; Wendt et al. 1994) which may also be interpreted as a regionally different response of alcohol on the brain metabolism. Wendt and Risberg (2001) and Wendt et al. (1994) proposed that ethanol causes a reduced inhibition of the left posterior cortex and a reduction of right-hemisphere information processing. Another explanation for regional differences may be the interaction of alcohol with neurotransmitters that are locally different distributed (Davies 2003; Little 1999; Yeh and Kolb 1997). However, we did not find such asymmetries which might indicate that Wendt's hypothesis may not easily be transferred to the motor system. It also shows that alcohol acts in a more complex way than just by a simple inhibition of regional $\mathrm{CBF}$ response or a reduction in the neuronal activity. While at moderate alcohol levels similar to those in the presented study simple repetitive movements will not suffer from a performance decrease, higher levels of alcohol or more complex movements will probably show more distortions reflecting the decrease of neuronal activation as well as the potential loss of the neurovascular coupling due to the increased alcohol-induced vasodilatation. It may even be speculated whether brain regions involved in emotional or higher cognitive functions may be more susceptible to ethanol and show distortions already at an earlier ethanol concentration which would explain why at moderate ethanol levels basic function such as walking or grasping are quite unaffected, while social behavior or internal emotional states are often already severely affected. 


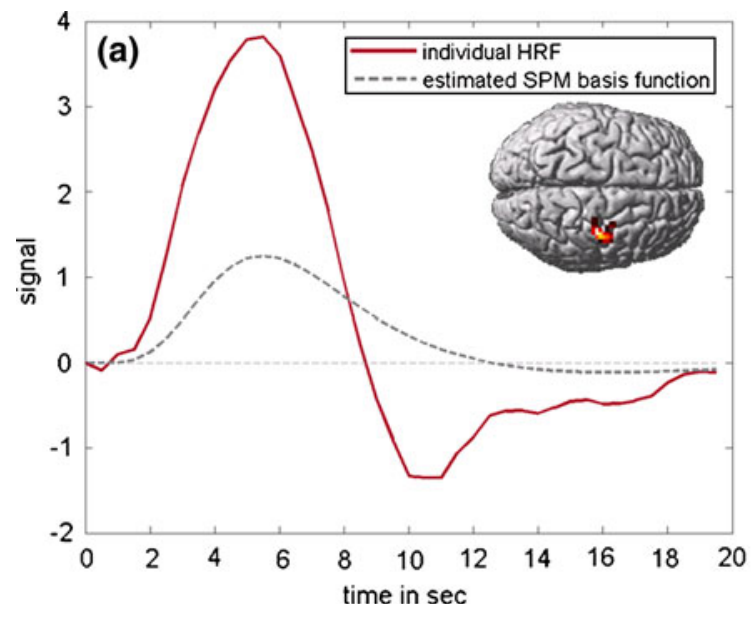

Fig. 3 Exemplary results of the standard statistical analysis with SPM before (left chart), and after (right chart) ethanol ingestion of one volunteer. The inlets depict the results of an event-related analysis of the RMC using the canonical SPM built-in HRF (FWE corr., $P<10^{-5}$ ) and WFU-Pickatlas (http://fmri.wfubmc.edu). The curves show absolute values of averaged HRF (after subtraction of mean baseline) and averaged beta values (as estimated by SPM) of the

Data analysis in the presence of altered BOLD signals

Despite the fact that the random-effects analysis using the GLM (Table 3) as well as the group analysis of the HRF form (Table 2) used the same data of the observed BOLDsignals both methods led to a contrary interpretation of the alcohol-induced effects.

While the GLM-based approach led to the conclusion that ethanol at moderate doses does not interfere with the BOLD-signal and thus has no effects on the neuronal activation the analysis of the alterations of the observed HRFs implies a strong influence of alcohol on the neurovascular coupling.

In order to analyze this discrepancy, the raw BOLDsignals of the observed regions were compared to the SPM built-in regressor multiplied by the estimated $\beta$-values. Fig. 3 shows an example where, despite a reduced amplitude of the HRF, the SPM-based general linear model analysis revealed an activation increase. Analyzing the data further showed that the reduced post-stimulus undershoot in the post-ethanol condition led to a stronger shift of the regressor function toward the positive overshoot. This resulted in a higher beta value that may be erroneously interpreted as an increased activation.

Depending on the shape of the time course of the observed HRF relative to the built-in regressor of the general linear model the statistical analysis of the other volunteers led to lowered, unchanged as well as increased $\beta$-values for the alcoholic condition when compared to the sober condition. These mixed patterns of activation

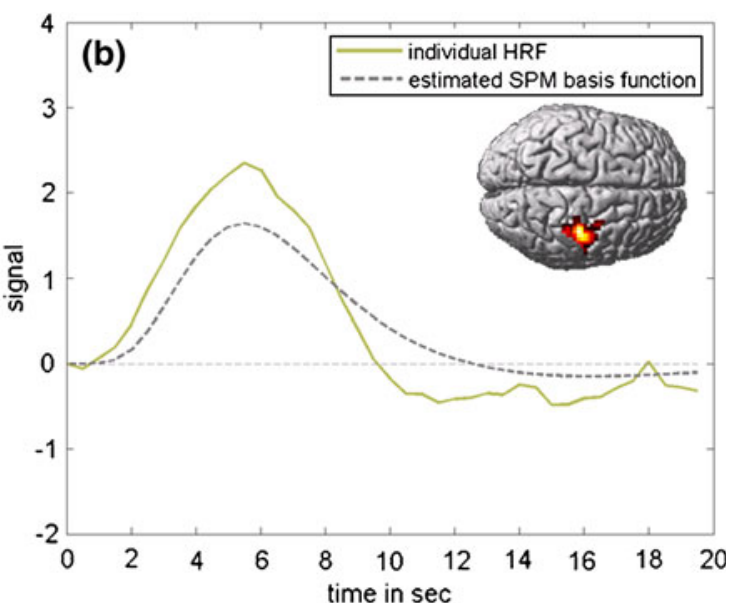

RMC. Solid lines averaged HRF; broken lines SPM-based estimation to the HRF using a double gamma-variate (basis function) as a regressor function multiplied with the beta value averaged across the ROI. The reduced post-stimulus undershoot in the post-ethanol condition leads to a stronger shift of the regressor function toward the positive overshoot. This results in a higher beta value that may be erroneously interpreted as an increased activation

changes may explain the false negative random effects group analysis.

The analysis of this potential pitfall of the statistical data analysis emphasizes a general problem for the reliability of pharmacological fMRI studies. In most pharmacological fMRI studies, the time course of the HRF is assumed to remain unchanged (Friston et al. 1995,2006) despite potential effects of experimental conditions or drugs on neurovascular coupling mechanisms. If the HRF changes its form considerably, this in turn may lead to false- positive or false-negative activation changes depending how the time course of the changed HRF correlates with a canonical HRF. Therefore, the direct analysis of the time course is important in experiments where changes of the HRF are to be expected. Characterizing the time course by appropriate parameters enables then a statistical analysis to gain insight into whether these parameters have changed significantly.

The effect of changed HRFs may be less obvious in most fMRI studies as the BOLD signal is often sampled more sparsely than in our study, especially if covering larger parts of the brain. Although potential HRF changes may then be less pronounced, pharmacological fMRI studies should still be tested for potential drug-induced changes to the HRF to avoid misleading interpretations of the statistical analysis, particularly if applying drugs in higher concentrations or examining drugs exhibiting strong vasoactive properties. This may explain why Van Horn et al. (2006) as well as Calhoun et al. (2004a) observed concomitant alcohol-induced decreases and increases using GLM-based approaches. 


\section{Conclusion}

Examining the impact of alcohol on the neurovascular coupling leads to complex alterations and different patterns depending on the cortical region. Instead of an isolated reduction of neuronal activation, the neurovascular coupling seems also to be affected. The altered hemodynamic response affects additionally the statistical fMRI data analysis leading to mis-interpretations in pharmacological fMRI. It is therefore recommended to determine potential individual drug-induced changes to the HRF.

Open Access This article is distributed under the terms of the Creative Commons Attribution Noncommercial License which permits any noncommercial use, distribution, and reproduction in any medium, provided the original author(s) and source are credited.

\section{References}

Aguirre GK, Zarahn E, D'Esposito M (1998) The variability of human, BOLD hemodynamic responses. NeuroImage 8(4):360-369

Blinkenberg M, Bonde C, Holm S, Svarer C, Andersen J, Paulson OB, Law I (1996) Rate dependence of regional cerebral activation during performance of a repetitive motor task: a PET study. J Cereb Blood Flow Metabol 16(5):794-803

Breiter HC, Gollub RL, Weisskoff RM, Kennedy DN, Makris N, Berke JD, Goodman JM, Kantor HL, Gastfriend DR, Riorden JP, Mathew RT, Rosen BR, Hyman SE (1997) Acute effects of cocaine on human brain activity and emotion. Neuron 19(3):591-611

Bruhn H, Kleinschmidt A, Boecker H, Merboldt K, Hanicke W, Frahm J (1994) The effect of acetazolamide on regional cerebral blood oxygenation at rest and under stimulation as assessed by MRI. J Cereb Blood Flow Metabol 14(5):742-748

Buxton RB, Wong EC, Frank LR (1998) Dynamics of blood flow and oxygenation changes during brain activation: the balloon model. Magn Reson Med 39(6):855-864

Calhoun VD, Altschul D, McGinty V, Shih R, Scott D, Sears E, Pearlson GD (2004a) Alcohol intoxication effects on visual perception: an fMRI study. Hum Brain Mapp 21(1):15-25

Calhoun VD, Pekar JJ, Pearlson GD (2004b) Alcohol intoxication effects on simulated driving: exploring alcohol-dose effects on brain activation using functional MRI. Neuropsychopharmacology 29(11):2097-2107

Chen YCI, Galpern WR, Brownell A, Matthews RT, Bogdanov M, Isacson O, Keltner JR, Beal MF, Rosen BR, Jenkins BG (1997) Detection of dopaminergic neurotransmitter activity using pharmacologic MRI: correlation with PET, microdialysis, and behavioral data. Magn Reson Med 38(3):389-398

Chung GH, Han YM, Jeong SH, Jack Jr CR (2005) Functional heterogeneity of the supplementary motor area. Am J Neuroradiol 26(7):1819-1823

Davies M (2003) The role of GABAA receptors in mediating the effects of alcohol in the central nervous system. J Psychiatry Neurosci 28(4):263-274

Fonseca LL, Alves PM, Carrondo MJT, Santos H (2001) Effect of ethanol on the metabolism of primary astrocytes studied by $13 \mathrm{C}$ and 31P-NMR spectroscopy. J Neurosci Res 66(5):803-811

Friston K, Ashburner J, Kiebel S, Nichols T, Penny W (2006) Statistical parametric mapping-the analysis of functional brain images. Academic Press, London
Friston KJ, Holmes AP, Poline J, Grasby PJ, Williams SCR, Frackowiak RSJ, Turner R (1995) Analysis of fMRI time-series revisited. NeuroImage 2(1):45-53

Friston KJ, Mechelli A, Turner R, Price CJ (2000) Nonlinear responses in fmri: the balloon model, volterra kernels, and other hemodynamics. NeuroImage 12(4):466-477

Glautier S, Remington B, Taylor C (1992) Alcohol placebos: you can only fool some of the people some of the time. Br J Addict 87(10): 1489

Hammersley R, Finnigan F, Millar K (1992) Alcohol placebos: you can only fool some of the people all of the time. $\mathrm{Br} \mathrm{J}$ Addict 87(10):1477-1480

Handwerker DA, Ollinger JM, D'Esposito M (2004) Variation of BOLD hemodynamic responses across subjects and brain regions and their effects on statistical analyses. NeuroImage 21(4):1639-1651

Hindmarch I, Kerr JS, Sherwood N (1991) The effects of alcohol and other drugs on psychomotor performance and cognitive function. Alcohol Alcohol 26(1):71-79

Horwitz B, Friston KJ, Taylor JG (2000) Neural modeling and functional brain imaging: an overview. Neural Netw 13(8-9):829-846

Jones AW, Jonsson KA (1994) Between-subject and within-subject variations in the pharmacokinetics of ethanol. $\mathrm{Br} \mathrm{J}$ Clin Pharmacol 37(5):427-431

Kaehkoenen S, Wilenius J, Nikulin VV, Ollikainen M, Ilmoniemi RJ (2003) Alcohol reduces prefrontal cortical excitability in humans: a combined TMS and EEG study. Neuropsychopharmacology Off Publ Am Coll Neuropsychopharmacol 28(4):747-754

Learn JE, Smith DG, McBride WJ, Lumeng L, Li T (2003) Ethanol effects on local cerebral glucose utilization in high-alcoholdrinking and low-alcohol-drinking rats. Alcohol 29(1):1-9

Levin JM, Ross MH, Mendelson JH, Kaufman MJ, Lange N, Maas LC, Mello NK, Cohen BM, Renshaw PF (1998) Reduction in BOLD fMRI response to primary visual stimulation following alcohol ingestion. Psychiatry Res Neuroimag 82(3):135-146

Lewis EG, Dustman RE, Beck EC (1970) The effects of alcohol on visual and somato-sensory evoked responses. Electroencephalogr Clin Neurophysiol 28(2):202-205

Little HJ (1999) The contribution of electrophysiology to knowledge of the acute and chronic effects of ethanol. Pharmacol Ther 84(3):333-353

Martin CS, Earleywine M, Finn PR, Young RD (1990) Some boundary conditions for effective use of alcohol placebos. J Stud Alcohol 51(6):500-505

Meda SA, Calhoun VD, Astur RS, Turner BM, Ruopp K, Pearlson GD (2009) Alcohol dose effects on brain circuits during simulated driving: an fMRI study. Human Brain Mapp 30(4):1257-1270

Moskowitz H, Burns MM, Williams AF (1985) Skills performance at low blood alcohol levels. J Stud Alcoh 46(6):482-485

Obata T, Liu TT, Miller KL, Luh W, Wong EC, Frank LR, Buxton RB (2004) Discrepancies between BOLD and flow dynamics in primary and supplementary motor areas: application of the balloon model to the interpretation of BOLD transients. NeuroImage 21(1):144-153

Ogawa S, Lee TM, Kay AR, Tank DW (1990) Brain magnetic resonance imaging with contrast dependent on blood oxygenation. Proc Natl Acad Sci USA 87(24):9868-9872

Oishi M, Mochizuki Y, Hara M, Takasu T (1995) P300 and xenon computed tomography before and after intravenous injection of acetazolamide. Arch Neurol 52(9):850-851

Rhodes LE, Obitz FW, Creel D (1975) Effect of alcohol and task on hemispheric asymmetry of visually evoked potentials in man. Electroencephalogr Clin Neurophysiol 38(6):561-568

Sano M, Wendt PE, Wirsen A, Stenberg G, Risberg J, Ingvar DH (1993) Acute effects of alcohol on regional cerebral blood flow in man. J Stud Alcohol 54(3):369-376 
Seidl S, Jensen U, Alt A (2000) The calculation of blood ethanol concentrations in males and females. Int J Legal Med 114(12):71-77

Seifritz E, Bilecen D, Hänggi D, Haselhorst R, Radü EW, Wetzel S, Seelig J, Scheffler K (2000) Effect of ethanol on BOLD response to acoustic stimulation-implications for neuropharmacological fMRI. Psychiatry Res Neuroimag 99(1):1-13

Seo DO, Rivier C (2003) Interaction between alcohol and nitric oxide on acth release in the rat. Alcohol Clin Exp Res 27(6):989-996

Shimosegawa E, Kanno I, Hatazawa J, Fujita H, Iida H, Miura S, Murakami M, Inugami A, Ogawa T, Itoh H, Okudera T, Uemura K (1995) Photic stimulation study of changing the arterial partial pressure level of carbon dioxide. J Cerebr Blood Flow Metabol 15(1):111-114

Stein EA, Pankiewicz J, Harsch HH, Cho J, Fuller SA, Hoffmann RG, Hawkins M, Rao SM, Bandettini PA, Bloom AS (1998) Nicotine-induced limbic cortical activation in the human brain: a functional MRI study. Am J Psychiatry 155(8):1009-1015

Stephan KE, Weiskopf N, Drysdale PM, Robinson PA, Friston KJ (2007) Comparing hemodynamic models with DCM. NeuroImage 38(3):387-401

Van Horn JD, Yanos M, Schmitt PJ, Grafton ST (2006) Alcoholinduced suppression of BOLD activity during goal-directed visuomotor performance. NeuroImage 31(3):1209-1221
Villringer A, Dirnagl U (1995) Coupling of brain activity and cerebral blood flow: basis of functional neuroimaging. Cerebrovasc Brain Metabol Rev 7(3):240-276

Volkow ND, Mullani N, Gould L, Adler SS, Guynn RW, Overall JE, Dewey S (1988) Effects of acute alcohol intoxication on cerebral blood flow measured with PET. Psychiatry Res 24(2):201-209

Volkow ND, Hitzemann R, Wolf AP, Logan J, Fowler JS, Christman D, Dewey SL, Schlyer D, Burr G, Vitkun S, Hirschowitz J (1990) Acute effects of ethanol on regional brain glucose metabolism and transport. Psychiatry Res Neuroimag 35(1):3948

Wendt PE, Risberg J (2001) Ethanol reduces rCFB activation of left dorsolateral prefrontal cortex during a verbal fluency task. Brain Lang 77(2): 197-215

Wendt PE, Risberg J, Stenberg G, Rosen I, Ingvar DH (1994) Ethanol reduces asymmetry of visual $\mathrm{rCBF}$ responses. J Cereb Blood Flow Metabol 14(6):963-973

Yeh HH, Kolb JE (1997) Ethanol modulation of GABA-activated current responses in acutely dissociated retinal bipolar cells and ganglion cells. Alcohol Clin Exp Res 21(4):647-655 